\title{
Manejo de la terapia anticoagulante durante el embarazo
}

\author{
María Angélica Durán-Morales* \\ Edwin J. Ariza-Parra** \\ Alejandro Colonia-Toro***
}

\begin{abstract}
* Residente de segundo año de Ginecología y Obstetricia. Universidad CES. Medellín. Antioquia. Colombia. **Médico Internista. Universidad de Antioquia, IPS Universitaria León XIII- Medellín. Antioquia. Colombia. ****édico ginecólogo y Obstetra. Universidad CES- Hospital General de Medellín. Antioquia. Colombia. Correspondencia: Dra. María Angélica Durán Morales. Dirección: Carrera 74\#53-55 apartamento 810, edificio Nuevo Mundo Los colores. Medellín.
\end{abstract} Antioquia. Colombia. Teléfono: 5778285. Correo electrónico: angelik0105@hotmail.com

Resumen

El embarazo se asocia con 5 a 10 veces mayor riesgo de enfermedad tromboembólica venosa, convirtiéndose en un escenario particular y complejo para la anticoagulación dada la necesidad de seguridad fetal y limitada representación en estudios de eficacia disponibles. Durante la gestación existen tres principales situaciones clínicas: la enfermedad tromboembólica venosa sin factor de riesgo precipitante, el síndrome antifosfolípido y las portadoras de válvulas mecánicas protésicas; todas con un alto riesgo de complicaciones materno-fetales, por lo que es vital conocer los anticoagulantes disponibles para cada patología, su evidencia científica, eventos adversos y características farmacológicas con el fin de realizar el mejor abordaje terapéutico y disminuir complicaciones asociadas. El objetivo es describir los avances de los anticoagulantes en escenarios clínicos de mayor impacto antes de la concepción, durante el embarazo y postparto y servir de herramienta con el fin de realizar el mejor abordaje terapéutico y disminuir complicaciones asociadas. MÉD.UIS.2020;33(2):65-73.

Palabras clave: Embarazo. Anticoagulantes. Prótesis valvulares cardíacas. Síndrome antifosfolípido. Tromboembolia.

\section{Management of anticoagulant therapy during pregnancy}

\begin{abstract}
Pregnancy is associated with a 5 to 10 -fold increased risk of venous thromboembolic disease, making it a complex setting for anticoagulation, given the need for fetal safety and limited representation in available efficacy studies. During pregnancy there are three main clinical situations: venous thromboembolic disease without a precipitating risk factor, antiphospholipid syndrome and prosthetic mechanical valve carriers; all with a high risk of maternal-fetal complications, so it is vital to know the anticoagulants available for each pathology, their scientific evidence, adverse events and pharmacological characteristics in order to perform the best therapeutic approach and reduce associated complications. The objective is to describe the advances of anticoagulants in clinical scenarios of greater impact before conception, during pregnancy and postpartum and to serve as a tool in order to carry out the best therapeutic approach and reduce associated complications. MÉD.UIS.2020;33(2):65-73
\end{abstract}

Keywords: Pregnancy. Anticoagulant. Mechanical prosthetic valves. Antiphospholipid syndrome. Thrombosis.

¿Cómo citar este artículo?: Durán-Morales MA, Ariza-Parra EJ, Colonia-Toro A. Manejo de la terapia anticoagulante durante el embarazo. MÉD.UIS.2020;33(2):65-73. doi: 10.18273/revmed.v33n22020007 


\section{Introducción}

El embarazo y el puerperio corresponden a periodos de alto riesgo trombótico; exacerbado por el compromiso de lo que se conoce como triada de Virchow con hipercoagulabilidad, lesión endotelial y estasis de flujo venoso, así como el incremento de los factores inhibidores de la fibrinólisis, los factores de la coagulación, fibrinógeno y dímero $\mathrm{D}$ en preparación a los estados de sangrado y la estasis vascular dado el aumento de cerca del 50\% del volumen plasmático, llevando finalmente a un desbalance en la hemostasia y la formación de trombos'.

Teniendo en cuenta los múltiples cambios fisiológicos que se dan en el puerperio y durante el embarazo, los eventos tromboembólicos pueden aumentar hasta 5-10 veces, y ser aún más frecuentes si presentan comorbilidades asociadas tales como preeclampsia, obesidad o sepsis durante la gestación ${ }^{2-5}$, haciendo que el embarazo constituya un periodo de alto riesgo para desarrollar patologías que sustancialmente pueden requerir terapia anticoagulante.

Los tres principales escenarios clínicos son: la enfermedad tromboembólica venosa, las trombofilias y las usuarias de válvulas mecánicas. En primer lugar la enfermedad tromboembólica venosa (ETV) que se define como un conjunto de alteraciones que comprenden la trombosis venosa profunda tanto de miembros superiores como inferiores, y el embolismo pulmonar. La incidencia de ETV aumenta a lo largo de todos los trimestres del embarazo, pero es más alta durante el período posparto'.

Las agencias de cooperación internacional redactan cada 15 años las razones de mortalidad materna, la última del 2015 muestra como el 14,9\% de muertes maternas a nivel mundial son relacionadas con los eventos tromboembólicos ${ }^{2}$. Siendo la principal causa de muerte materna en los países desarrollados o de altos ingresos, con una incidencia en reportes oficiales de alrededor de 0,76 a 1,72 por cada 1000 embarazos, de los cuales el 50\% ocurren antes de las 20 semanas de gestación. En los países de medianos y bajos ingresos los reportes no están tan claros dado que se da una tendencia a priorizar el reporte de las enfermedades más comunes como lo son la hemorragia postparto, los trastornos hipertensivos y la sepsis; tres eventos que pueden predisponer al desarrollo de enfermedades tromboembólicas, en donde el diagnóstico final de estas muertes podría ser sin lugar a duda el diagnóstico de tromboembolismo pulmonar no detectado ${ }^{3}$.

En Colombia, el perfil de las causas agrupadas de mortalidad materna muestran a los eventos tromboembólicos dentro de las 4 principales causas con un 3,6\%. Estas estadísticas colombianas han logrado mostrar grandes avances en cuanto a la reducción de la muerte materna; se evidencia una modificación en el perfil epidemiológico, según los reportes dados por el instituto nacional de salud en el 2015 los eventos tromboembólicos se empiezan a perfilar como una importante causa tanto directa como indirecta de muerte materna. Pero existe una población muy alta de pacientes que fallecen por una causa no determinada, y se sospecha que podrían estar relacionadas con esta patología ${ }^{7,8}$.

En segundo lugar; las trombofilias se dividen en heredadas y adquiridas; en donde la principal adquirida corresponde al síndrome antifosfolípido; una enfermedad autoinmune sistémica caracterizada por la presencia de trombosis venosa o arterial y/o pérdida del embarazo en presencia de anticuerpos antifosfolípidos positivos (anticoagulante lúpico, anticardiolipina y $\mathrm{B} 2$ glicoproteina) y el uso o no de anticoagulación en este grupo poblacional dependerá de la presencia o no de morbilidad fetal y/o trombosis asociada; en cuyo caso la elección del anticoagulante es por lo general heparina de bajo peso molecular ${ }^{9}$. Por último; pacientes usuarias de válvulas cardiacas protésicas principalmente mecánicas se asocian con un mayor riesgo tromboembólico durante el embarazo y la anticoagulación terapéutica se hace crucial para reducir el riesgo de este tipo de complicaciones; así como la elección del anticoagulante a usar que usualmente es warfarina si requiere de dosis bajas o heparina de bajo peso molecular de lo contrario como veremos más adelante ${ }^{10}$.

Las mujeres en el periodo del embarazo y/o postparto que van a requerir una terapia anticoagulante incluyen mujeres con alto riesgo de trombosis venosa profunda, mujeres con válvulas cardíacas protésicas, disfunción ventricular izquierda y algunas mujeres con pérdida fetal recurrente; siendo de suma importancia tener en cuenta la seguridad fetal y los problemas del periparto materno con el uso de los mismos (Inicio imprevisto del trabajo de parto, uso de anestesia neuroaxial, vía del parto, riesgo de sangrado); sin embargo, no se conocen estudios recientes en nuestro medio que nos aborden de 
una manera global e integral el mismo por lo que el objetivo de este artículo es realizar una revisión de los fármacos anticoagulantes, describir los avances en cuanto al uso y manejo de los mismos en cada uno en los principales escenarios clínicos durante la concepción, embarazo y postparto; así como dar a conocer los aspectos a tener en cuenta en las mujeres que reciben esta terapia, su seguridad durante la gestación y el papel que tiene cada uno en los diferentes escenarios clínicos más comunes durante estas etapas.

\section{Metodología de búsqueda}

La búsqueda se realizó en Pubmed y Cochrane con las palabras clave descritas: Embarazo, Anticoagulante, Válvulas protésicas mecánicas, Síndrome antifosfolípido, Tromboembolia, Trombosis. Teniendo como base artículos en español o inglés, publicados en los últimos 5 años (2013-2018). Se encontraron 324 artículos, de los cuales se excluyeron los duplicados y aquellos que no eran de acceso libre y según lo evidenciado en cada resumen. Se incluyeron revisiones sistemáticas, artículos originales, monografías y se escogieron los que permitían el acceso completo, actualizados, de cada uno de los temas escogidos tales como epidemiología, etiología, diagnóstico y tratamiento, quedando finalmente 45 referencias bibliográficas. (Ver Figura 1)

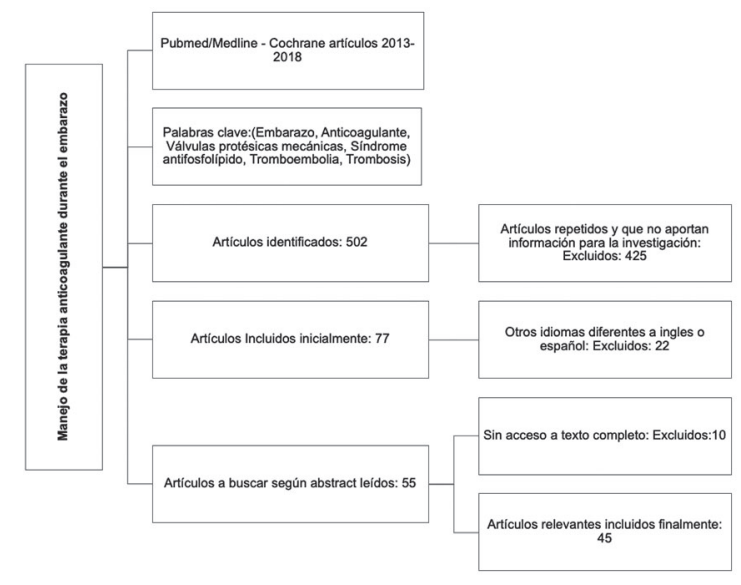

Figura 1. Flujograma de búsqueda

Fuente: autores.

\section{Desarrollo del tema}

\section{Anticoagulantes y su uso durante el embarazo}

La terapia anticoagulante es la intervención terapéutica farmacológica que actúa en cada fase de la hemostasia con el fin de corregir aquellas causas que desencadenen la tendencia trombótica. Según la FDA (Food and Drug Administration) los medicamentos administrados durante el embarazo y el puerperio tienen una clasificación de riesgo, este; compuesto por 5 categorias cada una con su letra, en ascenso según su riesgo estimado ( $A, B, C, D$ y $X$ ), siendo la categoría $A$ usualmente seguros en el embarazo, y los de la categoría X contraindicados por conocerse sus efectos deletéreos en el feto"1.

Estos anticoagulantes pueden dividirse según su mecanismo de acción, de tal forma que se cuentan con los siguientes ${ }^{1,12}$ (Ver Figura 2).

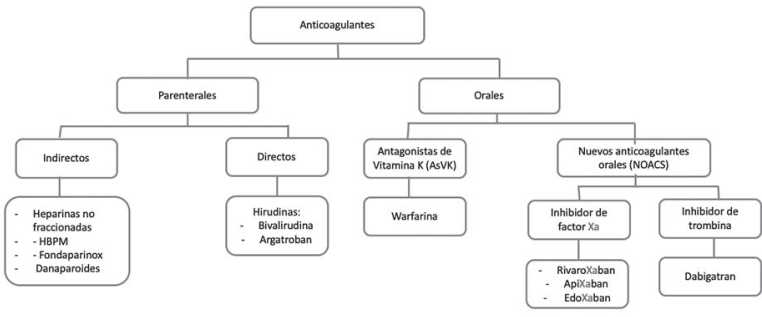

Figura 2. Clasificación de los anticoagulantes

Fuente: autores.

Los inhibidores indirectos como la heparina no fraccionada (HNF), heparina de bajo peso molecular (HBPM) y fondaparinox, tienen un efecto indirecto sobre la trombina; cuya actividad está mediada por cofactores plasmáticos y ejercen su actividad anticoagulante potenciando la antitrombina (un inhibidor endógeno de diversos factores de coagulación activados). Los anticoagulantes parenterales indirectos son los más estudiados en el embarazo y lactancia, y se catalogan como categoría $B$ (Estudios de reproducción en animales no han demostrado riesgo para el feto, no existen estudios controlados en embarazadas y se acepta su uso). Los tres anticoagulantes parenterales aprobados por la FDA, son Heparinas no fraccionadas, Heparinas de bajo peso molecular, fondaparinox y este último, usado solo en caso de contraindicación de heparina ${ }^{12-15}$. Las heparinas no cruzan la barrera fetoplacentaria ni se excretan a través de la leche materna, la HBPM tiene vida media variable, de 4 a 6 horas con 90-100\% de biodisponibilidad comparada con un $30 \%$ en la HNF. Los efectos adversos se pueden dividir en 4 categorias ${ }^{8,13-16}$ :

- Asociadas a sobredosis; la principal es el sangrado que dependiendo de la cantidad del mismo puede solucionarse desde la simple 
suspensión del medicamento hasta el uso de antídotos como el sulfato de protamina.

- Asociadas al uso prolongado; en donde por inducción de la reabsorción ósea acelerada, puede verse hasta después de 3 meses de uso, generando así osteoporosis.

- Asociados a formación de complejos inmunes: El cuadro se desarrolla luego de 5 días de tratamiento con heparina en hasta el $5 \%$ de los pacientes, y se puede e puede evitarse acortando el tiempo de su utilización al imbricar precozmente la infusión de heparina con los anticoagulantes orales.

- Asociado a impurezas en la mezcla en donde se puede presentar urticaria.

El uso de las HNF se reduce a aquellas pacientes con tasa de filtración glomerular menor a 30 o cuando hay un alto riesgo de sangrado que requiera una suspensión y reversión rápida de anticoagulación ${ }^{10}$.

Al ser más pequeñas que la heparina no fraccionada, las HBPM tienen menor unión celular y se depurán más lento, se absorben mejor por vía subcutánea y su unión a proteínas plasmáticas es menor, junto con una mejor dosis-respuesta. Por lo que se deduce su uso se prefiere ya que pueden usarse sin monitorización de laboratorio y tienen una relación dosis-respuesta más predecible ${ }^{13}$.

El seguimiento depende del mecanismo de acción, con HNF su monitoreo es con la medición del tiempo de tromboplastina parcial (60 a 85 segundos), mientras que con HBPM y en muy pocas ocasiones para el fondaparinox es con medición del factor anti Xa (valores normales 0.5 a $1 \mathrm{iu} / \mathrm{ml}$ ) debido a su mecanismo de acción basado principalmente en cofactores de forma indirecta; generalmente en aquellas que se encuentren en escenarios de alto riesgo trombótico ${ }^{13}$. El fondaparinox es de segunda linea en casos de hipersensibilidad grave a heparinas o Trombocitopenia inducida ${ }^{12,14-17}$.

- Inhibidores de la síntesis de las proteínas vitamina $K$ dependientes (warfarina): Si bien la warfarina es considerada categoría $D$ en el embarazo (evidencia de daño fetal en humanos, ocasiones en las que el beneficio supera los riesgos) ${ }^{11}$, este medicamento es especialmente útil en mujeres con alto riesgo trombótico como las usuarias de válvulas mecánicas cardíacas, en donde el beneficio de su uso es mayor al riesgo según la edad gestacional y dosis ${ }^{16}$. Los antagonistas de vitamina $\mathrm{k}$ (AVK) cruzan la barrera fetoplacentaria generando múltiples efectos en el feto (de predominio entre la semana 6-8 de gestación); dentro de los que se encuentran el llamado síndrome fetal cumarínico, que se caracteriza por microcefalia, hipoplasia mediofacial, epífisis punteadas hipoplasia de uñas y falanges (braquidactilia) entre otros como bajo peso al nacer, convulsiones, tono muscular bajo, dificultad para desarrollarse ${ }^{12-17}$. Se consideran dosis seguras las menores a 5mg/día durante el primer trimestre de embarazo o prenatal por lo que podrían usar el medicamento y para aquellas pacientes con alto riesgo trombótico y dosis más altas a estas ( >5mg/día) se considera realizar el cambio a HBPM. Durante las 3 semanas ante parto aquellas que tomen Warfarina deben pasar a HNF o HBPM para reducir el riesgo sangrado mayor por trauma durante el parto y hemorragia fetal $^{10,12,16,17}$.

- Los Inhibidores directos: (bivalirudina, argatroban) que no requieren cofactores de plasma para expresar su actividad, en donde su objetivo es la trombina. Y los inhibidores del factor Xa como el rivaroxabán, apixaban, edoxaban y el inhibidor de trombina, dabigatran, que corresponden a los nuevos anticoagulantes orales que no deben usarse durante el embarazo, o periodo de lactancia debido a mayores riesgos reproductivos en estudios con animales y datos no suficientes de seguridad en humanos ${ }^{18}$. El dabigatran y apixaban, cruzan de forma rápida la barrera fetoplacentaria generando concentraciones casi del 30-90\% comparadas con las concentraciones maternas, con riesgos evidenciados en animales de alteración en la fertilidad, reducción en implantación y sangrado ${ }^{19,20}$. En cuanto al rivaroxabán, los estudios en animales han mostrado: aborto postimplantación, disminución del peso corporal fetal y aumento en la resorción ósea; sin embargo, esto a dosis 4 veces mas alta que la usada en humanos. Aunque estudios recientes de calidad limitada sugieren seguridad en mujeres que se exponen inadvertidamente al inicio del embarazo, por ausencia de alteraciones fetales y neonatales; dado la falta de evidencia, se recomienda en aquellas que quedan en embarazo, cambiar el método de anticoagulación y realizar una evaluación ecográfica detallada para evaluar el desarrollo fetal ${ }^{21}$. 


\section{Mayo - agosto}

En la actualidad, es mayor el uso de nuevos anticoagulantes orales (NACOS) como alternativas más seguras que los AVK. Por esto las mujeres en edad fértil, deben tener vigilancia estrecha por la evidencia limitada en cuanto su uso durante lactancia y embarazo, por riesgos descritos de embriopatía y teratogenicidad ${ }^{18}$.

Ya conociendo cuales son los anticoagulantes disponibles, su mecanismo de acción y la seguridad en el embarazo entraremos en materia con los tres escenarios clínicos mas frecuentes que requieren anticoagulación:

\section{Embarazada con enfermedad tromboembólica venosa}

La enfermedad tromboembólica venosa se encuentra dentro de las principales causas de muerte en países en vía de desarrollo como Colombia, superado por los trastornos hemorrágicos, hipertensivos y la sepsis; mientras que en países desarrollados está dentro las 3 principales causas de muerte ${ }^{4-6}$. Generando tambien efectos en la morbilidad materna en donde tienen condiciones de difícil manejo tales como síndrome postrombótico que puede desarrollarse hasta en el $80 \%$ de los casos, y $60 \%$ insuficiencia venosa a los 5 años de seguimiento ${ }^{22}$. La incidencia es de 0,76 a 1,72 por cada 1000 embarazos presentándose la gran mayoría en el primer trimestre, y anteparto, con un riesgo de hasta del $33 \%$ en el puerperio (mas frecuente la embolia pulmonar) 5, $7,23-25$. La EP ocurre hasta en $25 \%$ de las TVP no tratadas, correspondiendo en el embarazo un $0.05 \%$ y una mortalidad asociada hasta del $15 \%^{24-28}$. Los factores que aumentan el riesgo son: edad mayor a 35 años, raza negra, obesidad y comorbilidades como: trombofilias, antecedente de trombosis, y síndrome antifosfolípido, los cuales aumentan el riesgo más de 15 veces ${ }^{22-24}$. El embarazo es un factor de riesgo independiente para enfermedad tromboembólica venosa, aun así, no se recomienda la anticoagulación terapéutica ni profiláctica de rutina, restringiéndola a eventos trombóticos o adicionales factores de riesgo para enfermedad tromboembólica venosa ${ }^{27-30}$.

La concientización alrededor de esta entidad es muy importante para poder hacer una presunción diagnostica y con esto un tratamiento empírico temprano y oportuno. Su diagnóstico en el embarazo es difícil, dado que sus síntomas son a menudo frecuentes durante la gestación como edema en miembros inferiores, disnea subjetiva y taquicardia.
Manejo de la terapia anticoagulante durante el embarazo

Por lo tanto, la sospecha basada en disnea súbita no explicada, hemoptisis y/o dificultad respiratoria de causa no definida deben ser hallazgos de alarma para iniciar el abordaje diagnóstico ${ }^{1,28,29}$. La mayor parte de los casos se producen en extremidades inferiores con predisposición de la extremidad inferior izquierda en el $88 \%$ secundario a la compresión anatómica de la vena iliaca izquierda y arterias iliacas y ováricas derechas que cruzan, así como la desviación uterina hasta este lado. En la mayoría se generan en venas proximales y $46 \%$ originados de las venas femorales e ilíacas ${ }^{31}$.

El tratamiento de elección son las heparinas, las cuales se inician tan pronto se sospecha clínicamente y se suspenden hasta que se descarte mediante pruebas objetivas ${ }^{29,30}$. La dosis recomendada de HBPM se debe ajustar según el peso cada 12 o 24 horas. En casos en que se requiera el uso de HNF por más de 5 días, se recomienda el recuento de plaquetas los días 4-14 y cada 72 horas con el fin de detectar eventos adversos ${ }^{1,27}$ (Ver Tabla 1).

Tabla 1. Características y dosis de las heparinas en enfermedad tromboembólica venosa

\begin{tabular}{|l|l|l|l|}
\hline Característica & HNF & HBPM & Fondaparinox \\
\hline Dosis & $-80 \mathrm{U} / \mathrm{Kg}$ bolo, & $1 \mathrm{~g} / \mathrm{Kg} \mathrm{c} / 12 \mathrm{hr}$ o & $5-10 \mathrm{mg} \mathrm{c} /$ \\
& $\begin{array}{l}18 \mathrm{U} / \mathrm{Kg} \mathrm{IV} \\
-150-200 \mathrm{U} / \mathrm{Kg} \\
\end{array}$ & c/24h & día SC \\
& $\mathrm{c} / 12 / \mathrm{hr} \mathrm{SC}$ & & \\
\hline Control & PTT & Anti Za & Raro Anti Xa \\
\hline Reversibilidad & Protamina & Protamina & No tiene \\
\hline
\end{tabular}

Fuente: adaptado de referencias 1, 12 y 27.

El parto por cesárea duplica el riesgo de enfermedad tromboembólica venosa, y el uso de terapia anticoagulante durante el mismo no aumento el sangrado e incluso llamativamente se asoció con menor porcentaje de infecciones de sitio operatorio, por esto no se debe retrasar la cesárea en caso de emergencia según su indicación obstétrica ${ }^{1,22}$. La anticoagulación durante el periparto se ha asociado a complicaciones hemorrágicas obstétricas hasta del $12,8 \%$, incluyendo aumento en hemorragia postparto (10\%) y hematomas perineales $(3 \%)^{32}$. Sin embargo, estos riesgos no contraindican su uso; se debe reducir los mismos con el cambio a Heparina no fraccionada y realizar suspensión previa al parto según vida media, con el fin de disminuir estos riesgos; así como conocer los tiempos necesarios para insertar o retirar un catéter para analgesia ${ }^{27,33}$ (Ver Tabla 2). 
Tabla 2. Tiempos entre la inserción y retiro del catéter para analgesia

\begin{tabular}{|c|c|c|}
\hline Heparina & $\begin{array}{l}\text { Intervalo para } \\
\text { inserción de catéter } \\
\text { epidural }\end{array}$ & $\begin{array}{l}\text { Intervalo para } \\
\text { reinicio de } \\
\text { coagulación }\end{array}$ \\
\hline HNF & $\begin{array}{l}\text { - >4 horas después } \\
\text { de parar la infusión } \\
\text { IV, TPT normal } \\
\text { - > } 12 \text { horas } \\
\text { después de } \\
\text { aplicación SC y TPT } \\
\text { normal }\end{array}$ & 1-8 horas \\
\hline HBPM & $>24$ horas & $\begin{array}{l}\text { - > } 24 \text { horas } \\
\text { después del inicio } \\
\text { de la analgesia } \\
->4 \text { horas después } \\
\text { del retiro del catéter }\end{array}$ \\
\hline
\end{tabular}

Fuente: adaptado de referencias 27 y 33.

La duración del tratamiento anticoagulante se debe dar según cada indicación durante el embarazo al menos 6 semanas postparto (mínimo $3-6$ meses de tratamiento). En el periodo de lactancia se puede continuar el tratamiento con warfarina y/o heparina ya que no se excretan en la leche materna y no inducen algún efecto anticoagulante en lactante ${ }^{34,35}$.

\section{Embarazada con trombofilias}

Las trombofilias se clasifican en adquiridas y heredadas ${ }^{36}$. Factores como la hipercoagulabilidad, una carencia de antitrombina III, proteína C o proteína S, son responsables del 20\% de los episodios tromboembólicos recurrentes. Se conoce que hasta en el $70 \%$ de los embarazos de pacientes con una deficiencia hereditaria de antitrombina III o déficit del factor $V$ de Leiden pueden presentar complicaciones tromboembólicas, y hasta un tercio de las que presentan déficit de proteína C, hiperhomocisteinemia y los anticuerpos antifosfolípidos persistentes ${ }^{36-38}$.

Todas estas patologías representan un alto riesgo de complicaciones neonatales como perdida gestacional temprana/tardía, preeclampsia y abruptio de placenta ${ }^{36,40}$. Es por esto, que existe indicación para tamización de trombofilias en pacientes con factores de riesgo como: pérdida gestacional recurrente, preeclampsia grave, historia de muerte in útero o RCIU de causa no explicada ${ }^{36-38}$.
Dentro de las trombofilias adquiridas, la patología más frecuentemente asociada corresponde al Síndrome de Anticuerpos Antifosfolípido (SAF), enfermedad autoinmune caracterizada por trombosis vascular y/o morbilidad del embarazo con presencia persistente de autoanticuerpos contra las proteínas de unión a los fosfolípidos (anticuerpos anticardiolipina, AntiB2 glicoproteína y/o anticoagulante lúpico). Así mismo, puede ser clasificada en primaria (sin evidencia clínica ni paraclínica de cursar con otra condición definida) o secundaria (infecciones, neoplasias, enfermedades autoinmunes u otros). La manifestación clínica más frecuente corresponde a la trombosis venosa (65-70\%). Estudios prospectivos evidencian que los fenómenos trombóticos se presentan en 5-12\% durante el embarazo y puerperio, aunque en algunas cohortes, se ha evidenciado hasta un $25 \%$ de casos. La trombosis arterial también se presenta en SAF y puede ocurrir en sitios atípicos: arteria retiniana y subclavia ${ }^{38-40}$.

Aunque los anticuerpos antifosfolípidos están clínicamente vinculados al SAF, no está claro si están involucrados en la patogénesis (se sabe que hasta el $5 \%$ de los individuos sanos tienen anticuerpos positivos), y de estos, < $<\%$ desarrollarán eventos trombóticos cada año ${ }^{40,41}$.

Para el diagnóstico, se han realizado consensos por expertos para establecer los criterios clínicos y de laboratorio para poder clasificar a las pacientes; por primera vez se describieron en 1999 (Sapporo), actualizados posteriormente en el 2006 (Sydney) que son los que son más tenidos en cuenta en la literatura, aumentando el diagnóstico a un 3-9\% comparados con los anteriores. Para su diagnóstico se requiere de al menos un criterio clínico y de laboratorio y que transcurra menos de 12 semanas o más de 5 años.9,39 (Ver Tabla 3).

En pacientes con SAF, se conocen factores de gravedad tales como: triple positividad, historia de evento trombótico, historia de trombosis y morbilidad obstétrica, altos títulos de anticuerpos anticardiolipina o B2 glicoproteína, así como la asociación con lupus. Se recomienda realizar una evaluación preconcepcional adecuada, ecografía mensual, ecografía Doppler desde la semana 28 cada 15 días, perfil biofísico semanal desde la semana 32 y a evaluar niveles de complemento cada trimestre ${ }^{9,38}$.

El tratamiento de estas pacientes se basa principalmente en si tiene o no antecedente de 
evento trombótico, en cuyo caso se recomienda el uso de dosis profiláctica de HBPM y/o ácido acetil salicílico, con el fin de reducir la pérdida del embarazo hasta en un $50 \%$ y eventos trombóticos un 40\%9,38 (Ver Figura 3). Desde un $15 \%$ de las pacientes con historia de perdida gestacional recurrente con anticuerpos positivos persistentes sin tratamiento hasta un 90\% tendrá nuevamente pérdida del embarazo. La combinación de HBPM + ASA a dosis bajas promueve la implantación del embrión en las etapas tempranas del embarazo, y además protege contra la trombosis de la vasculatura uteroplacentaria 9 ,38,41.

Tabla 3. Criterios Diagnósticos para SAF

\begin{tabular}{|c|c|}
\hline Criterios clínicos & Criterios de laboratorio \\
\hline $\begin{array}{l}\text { 1.Trombosis vascular } \\
1 \text { episodio o más de trombosis } \\
\text { (arterial o venosa) } \\
\text { 2. Morbilidad del embarazo } \\
\text { a) } 1 \text { o más abortos > semana } \\
10 \text { inexplicados de un feto } \\
\text { morfológicamente normal } \\
\text { b) } 1 \text { o más nacimientos } \\
\text { prematuros < semana } 34 \text { por } \\
\text { eclampsia o preeclampsia grave, } \\
\text { o características de insuficiencia } \\
\text { placentaria } \\
\text { c) } 3 \text { o más abortos espontáneos } \\
\text { consecutivos inexplicados < } \\
\text { semana } 10\end{array}$ & $\begin{array}{l}\text { 1. Anticoagulante lúpico (+) } \\
\text { en } 2 \text { o más ocasiones con } 12 \\
\text { semanas de diferencia } \\
\text { 2. Anticuerpo anticardiolipina } \\
\text { IgG y/o IgM en título alto (P99) } \\
\text { en } 2 \text { o más ocasiones con } 12 \\
\text { semanas de diferencia } \\
\text { 3. Anti B2 - Glucoproteína lgG } \\
\text { y/o IgM en título alto (P99) } \\
\text { en } 2 \text { o más ocasiones con } 12 \\
\text { semanas de diferencia }\end{array}$ \\
\hline
\end{tabular}

Fuente: Adaptado de Bulletins-Obstetrics, American \& Gynecologists, Obstetricians. (2012). Practice Bulletin No. 132: Antiphospholipid syndrome. Obstet Gynecol. 120. 1514-1521. 10.1097/01.AOG.0000423816.39542.0f.

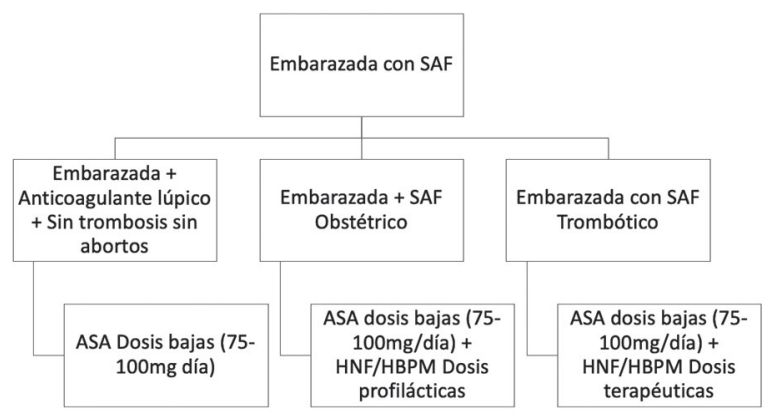

Figura 3. Esquemas de tratamiento en SAF Fuente: autores.

\section{Embarazada con enfermedad cardíaca valvular}

La enfermedad valvular corresponde a la principal causa de enfermedad cardíaca durante el embarazo en los países en desarrollo. La prevalencia de la misma es $<1 \%$, sin embargo, pueden aumentar el riesgo de eventos adversos maternofetales. Esta patología se asocia con alta tasa de morbimortalidad materna con requerimiento de anticoagulación permanente; un $42 \%$ tienen un parto complicado por arritmias, insuficiencia cardíaca, sangrado pre y postparto, complicaciones tromboembólicas e incluso la muerte por inadecuada anticoagulación ${ }^{10,16}$. La enfermedad cardíaca reumática es muy frecuente en los países en vía de desarrollo con una prevalencia de hasta el 90\% del total de las enfermedades cardíacas, siendo las usuarias de válvulas mecánicas las que necesitan de anticoagulación permanente por su alto riesgo trombótico. La evaluación preconcepcional en estas pacientes juega un papel importante, cuando la paciente ya está embarazada, se debe individualizar el tratamiento y discutir con la paciente las diferentes opciones de anticoagulación ${ }^{10,42}$. Existen múltiples esquemas de tratamiento ya estudiados; el primero se basa en el uso de warfarina sola (dosis bajas o altas), el segundo en el uso de heparinas durante todo el embarazo y por último el esquema secuencial (Heparina+ warfarina) $)^{10,43}$.

El uso de warfarina durante el embarazo se asocia a una menor complicación materna dado el alto riesgo trombótico en este grupo de pacientes, mientras que las heparinas son los más seguros para el feto. Dicho esto, se recomienda la warfarina para el segundo y tercer trimestre del embarazo, y su uso en el primer trimestre se considera en aquellos casos en donde la dosis diaria es $\leq 5 \mathrm{mg}$ (riesgo de embriopatía $<3 \%$ ). Con dosis $>5 \mathrm{mg} /$ día, se conocen eventos adversos fetales cinco veces mayores así como aborto espontáneo (26,7\%); por lo que es de suma importancia llegar a un acuerdo con la madre después de exponer los riesgos y beneficios del tratamiento ${ }^{42,43}$. El esquema de HNF en el primer trimestre, seguido por warfarina en el segundo y tercer trimestre, podría ser razonable para gestantes que reciben dosis altas de warfarina. Ninguno de estos tratamientos es inocuo; por su parte, el uso de heparinas puede evitar el riesgo de embriopatía, pero se asocia con incremento de trombosis valvular y embolia ${ }^{10,43}$. 
En la semana 36 de gestación se sugiere realizar el cambio a algún tipo de heparina con el fin de reducir la anticoagulación fetal, ya que la warfarina cruza la placenta; si el trabajo de parto se inicia sin haber podido realizar el cambio, se debe realizar parto por cesárea para disminuir el riesgo de sangrado cerebral fetal al pasar por el canal del parto ${ }^{10,17,42}$. Los niveles anti Xa se deben monitorizar y realizar 36 horas antes del parto en aquellas pacientes con HBPM, y en aquellos con HNF la infusión se suspenderá cuatro a seis horas antes del parto y se reiniciará nuevamente cuatro a seis horas después del mismo. Los niveles de antiXa recomendados para mantener su actividad varían dependiendo de las pautas que se prefieran siendo para la sociedad americana entre $0,8-1,2 \mathrm{u} / \mathrm{ml}^{44,45}$.

Se debe tener en cuenta que la gran mayoría de los estudios en los que se basan las recomendaciones son con evidencia de calidad baja a moderada, ya que para la gran mayoría de de las intervenciones se encontraron un número escaso de estudios, en particular por el hecho de que los estudios no son realizados en pacientes en gestación. Es necesario realizar más estudios de investigación para comparar directamente los fármacos sistémicos entre sí y así evaluar directamente los perfiles de seguridad.

\section{Conclusiones}

El embarazo constituye un factor de riesgo importante para el desarrollo de enfermedad tromboembólica, y mujeres en edad fértil están susceptibles a presentar diferentes patologías que requieran de anticoagulación profiláctica o terapéutica, sin embargo, estos no se indican de rutina. Se hace necesario recordar que las heparinas de bajo peso molecular son el medicamento de elección para y con mayor seguridad en la mayoría de los escenarios clínicos realizando la medición de niveles antiXa en pacientes específicos (insuficiencia renal crónica, obesidad). Por otro lado, todas las pacientes con prótesis mecánicas deben recibir anticoagulación terapéutica continua con monitoreos frecuentes. En aquellas con prótesis mecánicas que se suspenda la warfarina entre la semana 6 y 12 (dosis >5 mg/día), deben recibir HNF o HBPM con dosis ajustadas.

\section{Referencias bibliográficas}

1. James A. Practice Bulletin No. 123: thromboembolism in pregnancy. Obstet Gynecol. 2011;118(3):718-29.

2. OMS, UNICEF, UNFPA, Grupo del Banco Mundial y División de
Población de las Naciones Unidas, Trends in maternal mortality: 1990-2015, december 2015.

3. Ghaji N, Boulet SL, Tepper N, et al. Trends in venous thromboembolism among pregnancy-related hospitalizations, United States, 1994-2009. Am J Obstet Gynecol. 2013;209(5):433. e1-8.

4. Say L, Chou D, Gemmill A, Tunçalp Ö, Moller AB, Daniels J. Global causes of maternal death: a WHO systematic analysis. Lancet Glob Health. 2014;2(6):e323-33.

5. Grillo-Ardila CF, Amaya-Guío J, Molano-Franco D, Correa LP. Guía de práctica clínica para la prevención de eventos tromboembólicos venosos durante la gestación, el parto o el puerperio. Rev Colomb Obstet Ginecol. 2017;68(4):286-304.

6. Ministerio de salud y protección social. Análisis de situación de salud. Colombia. Diciembre. 2015.

7. Monroy AM, Martínez A, Montes de Oca D, Bolatti HE, Escobar MF. Guía clínica de la federación latino americana de sociedades de ginecología y obstetricia: enfermedad tromboembólica venosa y embarazo. FLASOG, GC;2014:1

8. Equipo Maternidad Segura Instituto Nacional de Salud Colombia. Vigilancia en salud pública de la maternidad segura. Sivigila. INS 2015.

9. Committee on Practice Bulletins-Obstetrics, American College of Obstetricians and Gynecologists. Practice bulletin no. 132: antiphospholipid syndrome. Obstet Gynecol. 2012;120(6):1514-21.

10. D'Souza R, Ostro J, Shah PS, Silversides CK, Malinowski A Murphy KE, et al. Anticoagulation for pregnant women with mechanical heart valves: a systematic review and meta-analysis. Eur Heart J. 2017; 38(19):1509-16.

11. Center for Drug Evaluation and Research. Evaluating the Risks of Drug Exposure in Human Pregnancies. U.S Department of Health and Human Services. Food and Drug Administration; 2005. http://www.fda.gov/downloads/Drugs/ GuidanceComplianceRegulatoryInformation/Guidances/ UCM071645.pdf

12. Garcia D, Baglin T, Weitz J, Samama M. Parenteral Anticoagulants. Chest. 2012;141(2): e24S-e43S.

13. Romualdi E, Dentali F, Rancan E. Anticoagulant therapy for venous thromboembolism during pregnancy: a systemic review and a meta-analysis of the literature. J Thromb Haemost. 2013; 11(2): 270-81.

14. Knol H, Schultinge L Erwich J, Meijer K. Fondaparinux as an alternative anticoagulant therapy during pregnancy. J Thromb Haemost. 2010;8(8):1876-9.

15. Greer JP, Arber DA, Glader B, List AF, Means RT, Rodgers GM. Wintrobe's clinical hematology. 13th ed. Wolters Kluwer; 2018.

16. Steinberg Z, Dominguez-Islas C, Otto C, Stout K, Krieger E. Maternal and Fetal Outcomes of Anticoagulation in Pregnant Women with Mechanical Heart Valves. J Am Coll Cardiol. 2017;69(22):2681-91.

17. Basu S, Aggarwal P, Kakani N, Kumar A. Low-dose maternal warfarin intake resulting in fetal warfarin syndrome: In search for a safe anticoagulant regimen during pregnancy. Birth Defects Res a Clin Mol Teratol. 2016; 106(2):142-7.

18. Beyer-Westendorf J, Michalski F, Tittl L, Middeldorp S, Cohen H, Abdul Kadir R, et al. Pregnancy outcome in patients exposed to direct oral anticoagulants - and the challenge of event reporting. Thromb and Haemost. 2016; 116(4):651-8.

19. Bapat P, Kedar R, Lubetsky A, Matlow JN, Aleksa K, Berger H, et al. Transfer of dabigatran and dabigatran etexilate mesylate across the dually perfused human placenta. Obstet Gynecol. 2014; 123(6): 1256-61.

20. Bapat P, Pinto LS, Lubetsky A, Aleksa K, Berger H, Koren G, et al. Examining the transplacental passage of apixaban using the dually perfused human placenta. Thromb and Haemost. 2016; 14(7): 1436-41.

21. Hoeltzenbein M, Beck E, Meixner K, Schaefer C, Kreutz R. Pregnancy outcome after exposure to the novel oral anticoagulant rivaroxaban in women at suspected risk for thromboembolic events: a case series from the German Embryotox Pharmacovigilance Centre. Clin Res Cardiol. 2016; 105(2): 117-26.

22. Blondon M, Casini A, Hoppe KK, Boehlen F, Righini M, Smith N. Risks of Venous Thromboembolism After Cesarean Sections: A Meta-Analysis. Chest. 2016;150(3):572-96

23. Alhassan S, Pelinescu A, Gandhi V, Naddour M, Singh A, Bihler E. Clinical Presentation and Risk Factors of Venous hromboembolic 
Disease. Crit Care Nurs Q. 2017;40(3):201-9.

24. Parunov LA, Soshitova NP, Ovanesov MV, Panteleev M, Serebriyskiy I. Epidemiology of venous thromboembolism (VTE) associated with pregnancy. Birth Defects Res C Embryo Today. 2015;105(3):167-84.

25. Gray G, Nelson-Piercy C. Thromboembolic disorders in obstetrics. Best Pract Res Clin Obstet Gynaecol. 2012;26(1):53-64.

26. Barco S, Nijkeuter M, Middeldorp S. Pregnancy and venous thromboembolism. Semin Thromb Hemost. 2013;39(5):549-58.

27. Chan WS, Rey E, Kent NE, Rey E, Corbett T, David M, et al. Venous thromboembolism and antithrombotic therapy in pregnancy. J Obstet Gynaecol Can. 2014; 36(6):527-53.

28. Konkle BA. Diagnosis and management of thrombosis in pregnancy. Birth Defects Res C Embryo Today. 2015;105(3):185-9.

29. Fogerty AE, Connors JM. Treating venous thromboembolism in pregnancy. Hematol Oncol Clin North Am. 2011;25(2):379-91.

30. Fukuda W, Chiyoya M, Taniguchi S, Daitoku K, Fukuda I. Management of deep vein thrombosis and pulmonary embolism (venous thromboembolism) during pregnancy. Thorac Cardiovasc Surg. 2016;64(6):309-14.

31. Chan WS, Spencer FA, Ginsberg JS. Anatomic distribution of deep vein thrombosis in pregnancy. Can Med Assoc J. 2010;182(7):65760 .

32. Limmer JS, Grotegut CA, Thames E, Dotters-Katz SK, Brancazio LR, James AH. Postpartum wound and bleeding complications in women who received peripartum anticoagulation. Thromb Res. 2013; 132(1): e19 - 23.

33. Butwick A, Hass C, Wong J, D Lyell , El-Sayed . Anticoagulant prescribing practices and anesthetic interventions among anticoagulated pregnant patients: a retrospective study. Int J Obstet Anesth. 2014;23(3):238-45.

34. Virkus RA, Løkkegaard EC, Lidegaard Ø, Langhoff-Roos J, Bjerregaard L, Skovlund CH, et al. Venous thromboembolism in pregnancy and the puerperal period: a study of 1210 events. Acta Obstet Gynecol Scand. 2013;92(10):1135-42.

35. Royal College of Obstetricians and Gynaecologists.
Thromboembolic disease in pregnancy and the puerperium: acute management. Royal College of Obstetricians and Gynaecologists. 2015; 37 (b).

36. Dobbenga-Rhodes Y. Shedding Light on Inherited Thrombophilias: The Impact on Pregnancy. J Perinat Neonatal Nurs. 2016;30(1):3644.

37. Ziakas PD, Poulou LS, Pavlou M, Zintzaras E. Thrombophilia and venous thromboembolism in pregnancy: a meta-analysis of genetic risk. Eur J Obstet Gynecol Reprod Biol. 2015; 191:106-11.

38. Arachchillage DRJ, Laffan M. Pathogenesis and management of antiphospholipid syndrome. Br J. Haematol. 2017;178(2):181195.

39. Antovic A, Sennström M, Bremme K, Svenungsson E. Obstetric antiphospholipid sindrome. Lupus Sci. Med. 2018;5(1):e000197.

40. Rezk M, Dawood R, Badr H. Maternal and fetal outcome in women with antiphospholipid syndrome: a three-year observational study. J Matern Fetal Neonatal Med. 2016;29(24):4015-9.

41. Rodrigues M, Afonso G, Kupty M, Bertassoni JC. Treatments and new perspectives of antiphospholipid syndrome in pregnancy. Medical Research. 2018;6(5).

42. Van Hagen IM, Roos-Hesselink JW, Ruys TP, Merz WM, Goland $\mathrm{S}$, Gabriel H, et al. Pregnancy in women with a mechanical heart valve. ROPAC. 2015;132(2):132-42.

43. $\mathrm{Xu} \mathrm{Z,} \mathrm{Fan} \mathrm{J,} \mathrm{Luo} \mathrm{X,} \mathrm{Zhang} \mathrm{WB,} \mathrm{Ma} \mathrm{J,} \mathrm{Lin} \mathrm{YB,} \mathrm{et} \mathrm{al.}$ Anticoagulation regimens during pregnancy in patients with mechanical heart valves: a systematic review and meta-analysis. Can J Cardiol. 2016;32(10): 1248.e1-1248.e9.

44. Goland S, Schwartzenberg S, Fan J, Kozak N, Khatri N, Elkayam U. Monitoring of anti-Xa in pregnant patients with mechanical prosthetic valves receiving low-molecular-weight heparin: peak or trough levels. J Cardiovasc Pharmacol Ther. 2014; 19(5):451.

45. Nishimura RA, Otto CM, Bonow RO, Carabello BA, ErwinIII JP, Fleisher LA, et al. Guideline for the management of patients with valvular heart disease: a report of the american college of cardiology/american heart association task force on practice guidelines. J Am Coll Cardiol. 2014; 63:e57. 